\section{Fortes prévalences sérologiques de l'anaplasmose bovine dans le Nord- Cameroun}

\author{
J.-Y. Chollet ${ }^{1 *}$
}

CHOLLET (J.-Y.). Fortes prévalences sérologiques de l'anaplasmose bovine dans le Nord-Cameroun. Revue Élev. Méd. vét. Pays trop., 1995, $48(1): 17-18$

De 1989 à 1994, les cas d'anaplasmose bovine aiguë ont rarement été diagnostiqués au Nord-Cameroun. Pourtant, le test d'agglutination rapide sur carte de Becton-Dickinson a permis de mettre en évidence des prévalences sérologiques élevées, surtout dans les provinces du Nord et de l'Extrême-Nord. L' anaplasmose bovine pourrait être cliniquement importante dans les régions où une plus grande proportion de bovins sont non immuns.

Mots clés : Bovin - Anaplasmose - Anaplasma marginale - Réaction d'agglutination - Epidémiologie - Cameroun.

Les prévalences sérologiques de l'anaplasmose bovine des zébus dans le Nord du Cameroun ont été évaluées grâce au test d'agglutination rapide sur carte (anaplasmosis card test) (1) de Becton-Dickinson. Le tableau I rapporte les échantillons étudiés et les résultats obtenus.
- dans le cadre d'un suivi bimensuel, réalisé pendant un an et demi sur des troupeaux sentinelles des provinces du Nord et de l'Extrême-Nord, les 79 frottis effectués sur les animaux présentant un taux d'hématocrite inférieur à 25 p. 100 n'ont permis de diagnostiquer qu'un seul cas d'anaplasmose clinique.

En revanche, les frottis lus à ces occasions ont plus fréquemment révélé des infections sub-cliniques ; ainsi, sur les 94 frottis du service de diagnostics du LANAVET évoqués précédemment, 12 ont été enregistrés comme permettant la mise en évidence d'Anaplasma marginale parasitant de faibles pourcentages d'hématies. Ce nombre de 12 est en fait en deçà de la réalité, la mention d'anaplamose n'ayant pas toujours été enregistrée lorsque la parasitémie était très faible. De même, la présence d'anaplasmose sub-clinique a été démontrée sur le troupeau de zébus du LANAVET grâce à un taurillon qui développa une anaplasmose aiguë après avoir subi une splénectomie ; cet animal mourut 33 jours après la splénectomie, avec 6 p. 100 de taux d'hématocrite.

Il est donc vraisemblable, au moins dans le Nord, et sans doute dans l'Extrême-Nord, qu'une forte proportion d'animaux soit infectée par $A$. marginale, mais qu'une immunité solide empêche, la plupart du temps, toute expression clinique de la maladie.

Tableau I

Prévalences sérologiques de l'anaplasmose bovine des zébus dans le Nord-Cameroun : échantillons étudiés et résultats obtenus

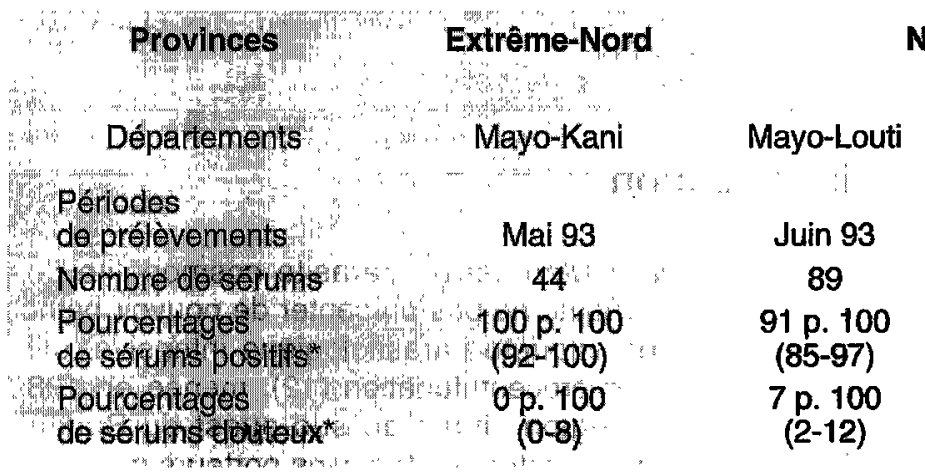

* Intervalles de confiance entre parenthèses.

Les prévalences sérologiques calculées sur ces échantillons sont élevées, par opposition aux cas d'anaplamose clinique (Anaplasma marginale) qui semblent rares :

- en 5 ans, les 94 frottis sanguins pratiqués par le service de diagnostics du LANAVET sur des animaux suspects d'hémoparasitose n'ont révélé des pourcentages d'hématies parasitées significatifs que pour 4 bovins, dont 3 dans la province de l'Adamaoua ;

1.Laboratoire national vétérinaire de Boklé, BP 503, Garoua, Cameroun.

* Adresse actuelle: DSV, 108 ave de la 1ère Armée française, 32020 Auch Cedex 9 , France.

Reçu le 21.3.1995, accepté le 11.4.1995.

\begin{tabular}{cc} 
Nord & Adamaoua \\
\hline Bénoue & Vina \\
Mai et Juin 93 & Juillet 93 \\
165 & 73 \\
94 p. 100 & 62 p. 100 \\
$91,4-97,6)$ & $(51-73)$ \\
3 p. 100 & 7 p. 100 \\
$(0,4-5 ; 6)$ & $(1-13)$
\end{tabular}
Ensemble des 3
provinces

Dans l'Adamaoua, la prévalence sérologique de l'anaplasmose semble plus faible et/ou plus hétérogène : 12 animaux positifs sur 30 du Centre de Recherches zootechnique et vétérinaire de Wakwa, et 33 positifs sur 43 bovins tout-venant de l'abattoir de Ngaoundéré. Les tailles de ces échantillons étant très faibles, il est difficile de conclure. Néanmoins, ces chiffres peuvent être rapprochés de l'hypothèse d'une plus grande fréquence des cas d'anaplasmose clinique dans cette province.

Une étude, portant sur un plus grand nombre de sérums de différentes localités de l'Adamoua, mais également d'autres provinces où l'élevage de bovins est important (Ouest, Nord-Ouest, Est), permettrait de vérifier l'hypothèse de prévalences hétérogènes et de préciser le risque épidémiologique d'anaplamose clinique. 


\section{Communications}

\section{Remerciements}

Le Heifer Project International nous a gracieusement fourni les kits de Becton-Dickinson (anaplasmosis card test). Le Dr F. Stachurski-Kühlman a réalisé pour nous les prélèvements de sang provenant de l'Adamaoua.

\section{Bibliographie}

1. AMERAULT T.E., ROBY T.O., 1968. A rapid card agglutination test for bovine anaplasmosis. J. Am. vet. Med. Assoc., 153: 1828-1834.

CHOLLET (J.-Y.). High serological prevalence rates of bovine anaplasmosis in Northern Cameroon. Revue Elev. Méd. vét. Pays trop., 1995, 48 (1): $17-18$

Between 1989 and 1994, cases of acute bovine anaplasmosis were rarely diagnosed in Northern Cameroon. However, the Becton-Dickinson anaplasmosis card test revealed high prevalence rates, especially in the North and Far North provinces. Bovine anaplasmosis might be clinically important in areas where the proportion of mon-innume cattle is higher.

Key words : Cattle - Anaplasmosis - Anaplasma marginale Agglutination test - Epidemiology - Cameroon.

\section{Absence d'interaction des infections à Trypanosoma theileri avec le diagnostic des trypanosomoses animales par détection des antigènes circulants}

\author{
A. Delafosse ${ }^{1}$ \\ Z. Bengaly ${ }^{1}$ \\ G. Duvallet ${ }^{1 *}$
}

DELAFOSSE (A.), BENGALY (Z.), DUVALLET (G.). Absence d'interaction des infections à Trypanosoma theileri avec le diagnostic des trypanosomoses animales par détection des antigènes circulants. Revue Elev. Méd. vét. Pays trop., 1995, 48 (1) : 18-20

Ce travail reprend des données accumulées au Centre international de Recherche-Développement sur l'Elevage en Zone subhumide (CIRDES) lors de suivis épidémiologiques. Les prévalences de Trypanosoma vivax, Trypanosoma congolense et Trypanosoma brucei obtenues à l'aide du test ELISA de détection des antigènes circulants ont été comparées chez des animaux infectés ou non par Trypanosoma theileri. Le but était de mettre en évidence l'existence d'éventuelles réactions sérologiques croisées entre $T$. theileri et les trypanosomes pathogènes. Les résultats obtenus montrent l'absence d'interaction des infections à $T$. theileri avec le diagnostic des trypanosomes pathogènes par détection des antigèncs circulants.

Mots clés : Trypanosomosc - Trypanosoma theileri - Tost ELISA - Diagnostic - Burkina Faso.

\section{Introduction}

Trypanosoma theileri est un parasite cosmopolite des Bovidés. II est réputé ne pas présenter de pouvoir pathogène (11). Une enquête parasitologique intensive, utilisant la double microcentrifugation (12), menée en 1989 au pays Lobi (région située au sud-ouest du Burkina Faso), a permis de détecter 52 bovins porteurs de ce trypanosome sur 188 prélevés (5):

La prévalence apparente de $T$. theileri dans cette zone était donc de 27 p. 100 alors qu'elle n'était que de 5,9 p. 100 pour l'ensemble des trypanosomes pathogènes. La'grande fréquence de $T$. theileri pose le problème des réactions croisées éventuelles avec les trypanosomes pathogènes ( $T$. vivax, $T$. congolense et $T$. brucel)

1. Centre international de Recherche-Développement sur l'Elevage en Zone subhumide (CIRDES), Unité Epidémiologie et Biotechnologie appliquée, $01 \mathrm{BP}$ 454, Bobo-Dioulasso 01, Burkina Faso.

* Adresse actuelle : CIRAD-EMVT, 10 rue Pierre Curie, 94704 Maisons-Alfort Cedex, France.

Reçu le 15.11.1994, accepté le 9.5.1995. 